\title{
ADAPTACIÓN PSICOMÉTRICA DEL CUESTIONARIO DE ESTILOS ATRIBUTIVOS
}

\section{PSYCHOMETRIC ADAPTATION OF THE QUESTIONNAIRE OF ATTRIBUTIONAL STYLES}

\author{
Maria Matalinares C. ${ }^{1}$, Rosa Tueros C., Juan Yaringaño L. \\ Universidad Nacional Mayor de San Marcos, Lima, Perú \\ (RECiBido el 2/02/2009, ACEPTADO el 11/06/2009)
}

\begin{abstract}
RESUMEN
El presente estudio tuvo como objetivo analizar la validez y confiabilidad del Cuestionario de Estilos Atributivos (CEA) de Alonso y Sánchez (1992). Se trabajó con 324 sujetos de 14 a 16 años, de ambos sexos, de $4 .^{\circ}$ y $5 .^{\circ}$ grado de educación secundaria. Los resultados demostraron que los puntajes obtenidos a través del CEA son confiables. Igualmente, se puede concluir que dicha prueba posee validez de constructo, ya que el criterio de jueces y las correlaciones de cada bloque con el módulo alcanzan valores significativos, la matriz de correlaciones reporta asociaciones significativas entre las áreas y el análisis factorial demuestra la existencia de dos factores.
\end{abstract}

Palabras clave: Cuestionario de estilos atributivos, adaptación, confiabilidad, validez.

\begin{abstract}
The present study had as objective to analyze the validity and reliability of the Questionnaire of Attributional Styles (QAS) of Alonso and Sanchez (1992). It was worked with 324 subjects from 14 to 16 years, of both sexes, of $4^{\circ}$ and $5^{\circ}$ degree of secondary education. The results demonstrated that scores obtained through the QAS are reliable. Equally, it is possible to conclude that the above mentioned test possesses construct validity, since judges' criteria and the correlations of each block with the module reach significant values, matrix of correlations reports significant associations between areas and the factorial analysis demonstrates existence of two factors.
\end{abstract}

Keywords: Questionnaire of attributional styles, adaptation, reliability, validity.

1 Docente investigadora del Instituto de Investigaciones Psicológicas de la Facultad de Psicología, Universidad Nacional Mayor de San Marcos, Lima-Perú. E-mail: maria_luisa93@hotmail.com 


\section{INTRODUCCIÓN}

De las teorías que se han propuesto para explicar la motivación humana en general y la motivación con que los alumnos afrontan el aprendizaje y el trabajo escolar en particular, se ha utilizado la Teoría Atribucional de Weiner (1974, 1986); Alonso y Mateos (1986). De acuerdo con esta teoría, se considera a la conducta como un continuo de episodios dependientes unos de otros, donde el éxito y el fracaso dan lugar, respectivamente, a respuestas emocionales positivas y negativas. Pero si los resultados son inesperados, como por ejemplo, aprobar cuando no se tenía esperanza de ello o desaprobar tanto si se esperaba como si no, tendemos a preguntarnos por las causas que los han determinado y a buscar respuesta a tales preguntas. Por ello es común oír con frecuencia entre los alumnos desaprobados comentarios como: "No sé por qué me han jalado", "Este profesor no me quiere", "El profesor se me ha prendido", y también comentarios de aquellos que no esperaban aprobar. "Qué suerte tengo" o "No sé cómo habré podido aprobar, estaba seguro que iba a jalar". Por otra parte, es importante recalcar que las explicaciones que damos influyen en nuestras respuestas emocionales y en nuestras expectativas y, a través de ellas, en el interés y esfuerzo que ponemos en conseguir alcanzar nuestras metas.

Ahora bien, Weiner (1986) subraya, como aportación novedosa, la importancia de las emociones suscitadas en la actividad dirigida a un fin, como determinantes del valor subjetivo de los fines. La motivación sería una secuencia histórica, donde la conducta humana es el resultado de una cadena de sucesos interrelacionados, desde los pensamientos, pasando por las atribuciones, las expectativas y las emociones, hasta llegar a la acción: la cadena empieza con el resultado de una conducta de logro ejecutada por un individuo que es juzgado como un éxito o un fracaso, según haya alcanzado, o no, el estándar. Este resultado provoca inmediatamente la aparición de un estado afectivo de felicidad, para el éxito percibido, y frustración o tristeza para el fracaso percibido (sentimientos dependientes del resultado), iniciándose una búsqueda causal para determinar la razón por la que se ha llegado al resultado. En esta búsqueda causal confluyen numerosos antecedentes, fundamentalmente, la historia de logro pasada del sujeto, el rendimiento de los demás, etc. que permiten alcanzar una atribución causal del resultado; la atribución causal percibida desencadena nuevas emociones (dependientes de la atribución), y ambas, causalidad y emociones, contribuyen a determinar la conducta futura.

Puede considerarse que existen tantos tipos de estilos atributivos como causas a las que atribuir los hechos que se tratan de explicar. Las distintas causas a las que pueden atribuirse los hechos observados en relación con la propia conducta pueden clasificarse dentro de distintas dimensiones: interno-externo, estable-variable, controlable-no controlable, general-específico (Weiner, 1979, 1986; Alonso Tapia, 1983); los estilos atributivos pueden definirse, en primer lugar, en función de tales dimensiones causales, con las implicaciones que esto conlleva cuando se trata de predecir el comportamiento del sujeto.

\section{MÉTODO}

La presente investigación es de tipo aplicada, con método correlacional comparativo, diseño transversal (Hernández, R. y otros 2003). 


\section{Sujetos}

El universo de la investigación estuvo conformado por los alumnos de 4to y 5to. de secundaria de instituciones educativas nacionales de Lima Metropolitana. Para la obtención de la muestra se llevó a cabo un muestreo probabilístico. La muestra total inicial fue de 394 alumnos, pero en vista que 66 alumnos no contestaron todas la preguntas, se eliminaron los cuestionarios. Quedando solo 324 alumnos, de ambos sexos, cuyas edades fluctuaban entre los 14 y 16 años.

Tabla N.$^{\circ}$ 1. Distribución de la muestra según grado de estudio y sexo.

\begin{tabular}{ccccc}
\hline \multicolumn{2}{c}{$\mathbf{4}^{\circ}$ grado } & \multicolumn{3}{c}{$\mathbf{5}^{\circ}$ grado } \\
\hline Masculino & Femenino & Masculino & Femenino & Total \\
\hline \multirow{2}{*}{85} & 75 & 84 & 80 & 324 \\
\hline
\end{tabular}

\section{Instrumento}

En la investigación se utiliza el Cuestionario de Estilos Atributivos (EAT) propuesto por Alonso y Sánchez (1992). Para establecer la confiabilidad, los autores utilizaron el método de consistencia interna. Los coeficientes de fiabilidad en el Área de Logros Académicos son para la escala I de 0,84, la Escala II 0,76 y para la Escala III 0,711; en el Área de Relaciones Interpersonales, en la Escala I es de 0,81 y para la Escala II 0,77. En el método de consistencia interna por división por mitades, obtuvieron una correlación de Pearson de 0,74 y en la fórmula de corrección de Spearman-Brown de 0,72. La validez de contenido la realizaron por un proceso de validación por jueces. Los resultados del coeficiente $\mathrm{V}$ de Aiken señalan que los ítemes alcanzan coeficientes iguales o mayores que 0,78 con lo que tiene una probabilidad asociada de 0,48 o menos, los cuales fueron aceptados por los jueces.

El cuestionario es de aplicación colectiva e individual, en un tiempo aproximado de 45 minutos, con el propósito de evaluar los patrones atributivos en el ámbito de las relaciones interpersonales y de los logros académicos.

Esta prueba está constituida por las siguientes áreas:

\section{1) Logros académicos:}

- Externalizacion e incontrolabilidad de los resultados académicos. Hacen referencia a la atribución de los éxitos académicos a causas externas al propio sujeto no controlables por él, a saber, la suerte y otras personas.

- Atribución del fracaso académico a la falta de esfuerzo. Hace referencia al fracaso académico, resultado que se atribuye a la falta de esfuerzo, causa normalmente percibida como interna, variable y controlable,

- Atribución del éxito académico a la habilidad. En estos elementos el éxito se atribuye a causas internas, estables y controlables. 
- Atribución del fracaso al profesor. El contenido de los elementos hace referencia a la atribución del fracaso al profesor. Al igual que en otros factores, incluye todos los elementos generales y específicos que se esperaba que estuviesen relacionados. Este factor se percibe como causa normalmente externa y no controlable.

- Atribución del éxito al esfuerzo, externalizacion e incontrolabilidad del fracaso académico por su atribución a la mala suerte. Causa habitualmente percibida como interna, variable y controlable.

- Atribución del fracaso a la falta de habilidad. El fracaso académico se atribuye a la falta de habilidad, causa normalmente percibida como interna, estable y no controlable.

\section{2) Relaciones interpersonales}

- Internalización del fracaso en las relaciones interpersonales. Hacen referencia a la atribución fracaso en las relaciones interpersonales a causas internas -falta de esfuerzo o de habilidad.

- Atribución del éxito en las relaciones interpersonales al esfuerzo. Hacen referencia a la atribución del éxito en las relaciones interpersonales al esfuerzo, éste es el nombre que hemos dado a este factor.

- Externalizacion del éxito en las relaciones interpersonales. En estos elementos el fracaso se atribuye a la falta de habilidad y el éxito se atribuye a la misma causa o a causas externas como la suerte o los otros (resto de los elementos).

- Atribución del éxito en las relaciones interpersonales a la habilidad. El contenido de los elementos hace referencia a la atribución del éxito en las relaciones interpersonales a la habilidad y a las otras personas. Refleja la tendencia a internalizar el éxito, que se atribuye a la habilidad.

- Externalizacion del fracaso. El contenido de los mismos hace referencia a la atribución del fracaso a la mala suerte o a acción de otras personas, esto es, a causas externas.

\section{Validez de contenido del Cuestionario de Estilos Atributivos}

Para verificar la validez, se hizo una revisión detallada del contenido de los ítemes, con el objetivo de determinar si el lenguaje utilizado se adecuaba al lenguaje estándar de nuestro medio. Se propuso las modificaciones lingüísticas a los ítemes originales de la prueba, que fueron sometidas a criterio de jueces.

Se seleccionó un grupo de ocho jueces expertos en el campo del lenguaje, a quienes se les entregó una ficha con los ítemes originales solicitándoles la aprobación o desaprobación de estos. Para ello se utilizó el Coeficiente V. De Aiken, el cual, de acuerdo con Escurra (1988), requiere de un grupo de ocho jueces y que por lo menos siete estén en concordancia para que el ítem sea válido, alcanzando coeficiente $\mathrm{V}$ iguales o superiores a 0.88 , a un nivel de significación estadística de $\mathrm{p}<0.05$ 
En segundo lugar se estableció la confiabilidad en términos generales, entendida como el grado de consistencia que existe entre dos medidas de un mismo objeto (Anastasi, 1998). Para el presente estudio, se utilizó el método de consistencia interna con el propósito de evaluar el grado en que los ítemes del test están relacionados con el puntaje total. Esta forma de confiabilidad se caracteriza porque se obtiene a partir de una sola aplicación de la prueba, lo cual es útil en el caso de medir rasgos o características motivacionales. La estimación de la consistencia interna se realizó mediante la separación por mitades, es la división del instrumento en dos partes iguales, ya sean pares o impares. Luego de esta partición se procede a correlacionar los resultados para, posteriormente, utilizando la fórmula profética de Spearman, estimar la confiabilidad de la prueba completa.

\section{RESULTADOS}

\section{Validez de contenido}

El análisis de la aprobación-desaprobación de las modificaciones realizadas a los ítemes del Cuestionario de Estilos Atributivos, ha sido establecido a través del método de criterio de jueces utilizando el coeficiente $\mathrm{V}$ de Aiken (tabla N. ${ }^{\circ}$ ), obteniéndose los siguientes resultados:

- En el Área de logros académicos, constituida por la Escala 1, Externalización e incontrolabilidad de los resultados académicos y fundamentalmente el éxito, se obtuvo que de los 8 ítemes que conforman este factor, 5 de ellos presentan una $\mathrm{V}$ de 1,00, y 3 ítems alcanzaron una V. de 0,88; hallándose una V. total de 0,94. Escala 2, Atribución del fracaso académico a la falta de esfuerzo, se obtuvo que de los 6 ítemes que conforman este factor, 1 de ellos presenta una $\mathrm{V}$ de 1,00; 4 ítemes alcanzan una $\mathrm{V}$. de 0,88 , y 1 ítem tiene una V; hallándose una V. total de 0,50; Escala 3, Atribución del éxito académico a la habilidad. Se obtuvo que de los 6 ítemes que conforman este factor, 4 de ellos presentan una $\mathrm{V}$ de 1,00 y 2 ítems alcanzaron una $\mathrm{V}$. de 0,88; hallándose una V. total de 0,96 . Escala 4, Atribución del fracaso al profesor, se obtuvo que de los 6 ítemes que conforman este factor, 5 de ellos presentan una $\mathrm{V}$ de 1,00, mientras que 1 ítem tiene una V. de 0,88 ; hallándose una V. total de 0,98. Escala 5, Atribución del éxito al esfuerzo; se obtuvo que de los 6 ítemes que conforman este factor, 4 de ellos presentan una $\mathrm{V}$ de 1,00, mientras que 2 ítemes alcanzaron una $\mathrm{V}$. de 0,88 ; hallándose una $\mathrm{V}$. total de 0,96 . Escala 6 , Externalización e incontrolabilidad del fracaso académico por su atribución a la mala suerte, se obtuvo que de los 5 ítemes que conforman este factor, 2 de ellos presentan una $\mathrm{V}$ de 1,00 , mientras que 3 ítemes logran una V. de 0,88; hallándose una V. total de 0,93. Escala 7, Atribución del fracaso a la falta de habilidad, se obtuvo que de los 4 ítemes que conforman este factor, 2 de ellos presentan una $\mathrm{V}$ de 1,00, mientras que 2 ítems alcanzaron una $\mathrm{V}$. de 0,88 ; hallándose una $\mathrm{V}$. total de 0,94 .

- En el Área de las relaciones interpersonales. Constituida por la Escala 8, Internalización del fracaso en las relaciones interpersonales, se obtuvo que de los 7 ítemes que conforman este factor, 3 de ellos presentan una V de 1,00; mientras 
que 4 ítemes alcanzaron una $\mathrm{V}$. de 0,88 , hallándose una $\mathrm{V}$. total de 0,93. Escala 9, Atribución del éxito en las relaciones interpersonales al esfuerzo, se obtuvo que de los 6 ítemes que conforman este factor, 5 de ellos presentan una $\mathrm{V}$ de 1,00; mientras que 1 ítem presenta una $\mathrm{V}$. de 0,88 , hallándose una V. total de 0,98. Escala 10, Externalizacion del éxito en las relaciones interpersonales, se obtuvo que de los 6 ítems que conforman este factor, 2 de ellos presentan una $\mathrm{V}$ de 1,00, mientras que 4 ítemes alcanzaron una V. de 0,88, hallándose una V. total de 0,92. Escala 11, Atribución del éxito en las relaciones interpersonales a la habilidad, se obtuvo que de los 5 ítemes que conforman este factor, 2 de ellos presentan una $\mathrm{V}$ de 1,00; mientras que 3 ítemes alcanzaron una V. de 0,88, hallándose una V. total de 0,93. Escala 12, Eexternalizacion del fracaso; se obtuvo que de los 7 ítemes que conforman este factor, 4 de ellos presentan una $\mathrm{V}$ de 1,00, mientras que 2 ítemes alcanzaron una $\mathrm{V}$. de 0,88 , hallándose una $\mathrm{V}$. total de 0,95 .

Tabla N. ${ }^{\circ}$ 2. Validez de contenido por criterio de jueces del Cuestionario de Estilos Atributivos.

\begin{tabular}{|c|c|c|c|c|c|c|c|}
\hline \multicolumn{4}{|c|}{ Área de logros Académicos } & \multicolumn{4}{|c|}{ Área de las Relaciones Interpersonales } \\
\hline & Ítem & V Aiken & Decisión & & Ítem & V Aiken & Decisión \\
\hline \multirow{8}{*}{$\begin{array}{l}\text { Escala 1: } \\
\text { Externalizacion e } \\
\text { incontrolabilidad } \\
\text { de los resultados } \\
\text { académicos y } \\
\text { fundamentalmente } \\
\text { el éxito }\end{array}$} & 20 & 1,00 & A & \multirow{8}{*}{$\begin{array}{l}\text { Escala 8: } \\
\text { internalización } \\
\text { del fracaso en } \\
\text { las relaciones } \\
\text { interpersonales }\end{array}$} & 10 & 0,88 & A \\
\hline & 33 & 1,00 & A & & 16 & 1,00 & A \\
\hline & 40 & 0,88 & A & & 27 & 0,88 & A \\
\hline & 51 & 0,88 & A & & 29 & 1,00 & A \\
\hline & 52 & 1,00 & A & & 54 & 0,88 & A \\
\hline & 56 & 0,88 & A & & 70 & 0,88 & A \\
\hline & 62 & 1,00 & A & & 71 & 1,00 & A \\
\hline & 64 & 1,00 & A & & & & \\
\hline \multirow{6}{*}{$\begin{array}{l}\text { Escala 2: } \\
\text { Atribución del } \\
\text { fracaso académico a } \\
\text { la falta de esfuerzo }\end{array}$} & 7 & 1,00 & A & \multirow{6}{*}{$\begin{array}{l}\text { Escala 9: } \\
\text { atribución del éxito } \\
\text { en las relaciones } \\
\text { interpersonales al } \\
\text { esfuerzo }\end{array}$} & 4 & 1,00 & A \\
\hline & 17 & 0,88 & A & & 12 & 1,00 & A \\
\hline & 26 & 0,88 & A & & 31 & 1,00 & A \\
\hline & 34 & 0,88 & A & & 38 & 0,88 & A \\
\hline & 47 & 0,88 & A & & 55 & 1,00 & A \\
\hline & 72 & 0,50 & $\mathrm{R}$ & & 66 & 1,00 & A \\
\hline \multirow{6}{*}{$\begin{array}{l}\text { Escala 3: } \\
\text { Atribución del éxito } \\
\text { académico a la } \\
\text { habilidad. }\end{array}$} & 2 & 1,00 & A & \multirow{6}{*}{$\begin{array}{l}\text { Escala 10: } \\
\text { externalizacion } \\
\text { del éxito en } \\
\text { las relaciones } \\
\text { interpersonales. }\end{array}$} & 1 & 1,00 & A \\
\hline & 14 & 1,00 & A & & 15 & 0,88 & A \\
\hline & 23 & 0,88 & A & & 24 & 0,88 & A \\
\hline & 44 & 1,00 & A & & 41 & 0,88 & A \\
\hline & 59 & 0,88 & A & & 45 & 1,00 & A \\
\hline & 60 & 1,00 & A & & 61 & 0,88 & A \\
\hline
\end{tabular}

continúa 
María Matalinares C., Rosa Tueros C., Juan Yaringaño L..

\begin{tabular}{|c|c|c|c|c|c|c|c|}
\hline \multicolumn{4}{|c|}{ Área de logros Académicos } & \multicolumn{4}{|c|}{ Área de las Relaciones Interpersonales } \\
\hline & Ítem & V Aiken & Decisión & & Ítem & V Aiken & Decisión \\
\hline \multirow{6}{*}{$\begin{array}{l}\text { Escala 4: Atribu- } \\
\text { ción del fracaso al } \\
\text { profesor }\end{array}$} & 6 & 1,00 & A & \multirow{6}{*}{$\begin{array}{l}\text { Escala 11: atri- } \\
\text { bución del éxito } \\
\text { en las relaciones } \\
\text { interpersonales a la } \\
\text { habilidad. }\end{array}$} & 18 & 0,88 & A \\
\hline & 9 & 0,88 & A & & 25 & 1,00 & A \\
\hline & 28 & 1,00 & A & & 39 & 0,88 & A \\
\hline & 36 & 1,00 & A & & 48 & 1,00 & $\mathrm{~A}$ \\
\hline & 53 & 1,00 & A & & 67 & 0,88 & A \\
\hline & 69 & 1,00 & A & & & & \\
\hline \multirow{7}{*}{$\begin{array}{l}\text { Escala 5: } \\
\text { Atribución del éxito } \\
\text { al esfuerzo. }\end{array}$} & 3 & 1,00 & A & \multirow{7}{*}{$\begin{array}{l}\text { Escala 12: } \\
\text { Externalizacion del } \\
\text { fracaso }\end{array}$} & 22 & 1,00 & A \\
\hline & 13 & 0,88 & A & & 32 & 0,88 & A \\
\hline & 21 & 1,00 & A & & 35 & 0,88 & A \\
\hline & 42 & 1,00 & A & & 43 & 1,00 & A \\
\hline & 57 & 0,88 & A & & 46 & 1,00 & A \\
\hline & 63 & 1,00 & A & & 50 & 0,88 & A \\
\hline & & & & & 58 & 1,00 & $\mathrm{~A}$ \\
\hline \multirow{5}{*}{$\begin{array}{l}\text { Escala 6: } \\
\text { Externalizacion e } \\
\text { incontrolabilidad del } \\
\text { fracaso académico } \\
\text { por su atribución a } \\
\text { la mala suerte. }\end{array}$} & 5 & 1,00 & A & & & & \\
\hline & 11 & 0,88 & A & & & & \\
\hline & 30 & 0,88 & A & & & & \\
\hline & 37 & 0,88 & A & & & & \\
\hline & 65 & 1,00 & A & & & & \\
\hline \multirow{4}{*}{$\begin{array}{l}\text { Escala 7: } \\
\text { Atribución del } \\
\text { fracaso a la falta de } \\
\text { habilidad }\end{array}$} & 8 & 1,00 & A & & & & \\
\hline & 19 & 0,88 & A & & & & \\
\hline & 49 & 0,88 & A & & & & \\
\hline & 68 & 1,00 & A & & & & \\
\hline
\end{tabular}

\section{Confiabilidad del cuestionario}

En la tabla $\mathrm{N}^{\circ} 3$ se presentan los resultados del análisis de las escalas Cuestionario de Estilos Atributivos, con la media y la correlación ítem-test corregida, asumiéndose los puntajes de cada bloque como si fueran un ítem. Igualmente se presenta el coeficiente alpha de Cronbach como medida de confiabilidad. El criterio para aceptar o rechazar la correlación ítem-test corregida es que debe ser mayor o igual a $=, 20$ (Nunnaly \& Bernstein, 1995). Las correlaciones ítem-test oscilan entre 0,25 y 0,51 observándose que todos los bloques obtienen correlaciones significativas con el puntaje total de módulo, siendo las escalas más discriminativas la Escala 1, Externalizacion e incontrolabilidad de los resultados académicos $(0,51)$ y la escala 3 , Atribución del éxito académico a la habilidad $(0,84)$. El alpha de Cronbach calculado para el Cuestionario alcanza un valor de 0,662 de manera que se puede decir que el Cuestionario de Estilos Atributivos nos permite obtener puntajes confiables. 
Tabla N.$^{\circ}$ 3. Índice de correlación ítem-test corregido del Cuestionario de Estilos Atributivos.

\begin{tabular}{ccccc}
\hline Escala & $\mathbf{M}$ & $\begin{array}{c}\text { Varianza de Escala si } \\
\text { ítem es eliminado }\end{array}$ & $\begin{array}{c}\text { Correlación Ítem- } \\
\text { Total Corregida }\end{array}$ & $\begin{array}{c}\text { Alpha de Cronbach si } \\
\text { ítem es eliminado }\end{array}$ \\
\hline 1 & 130,26 & 419,468 &, 511 &, 599 \\
2 & 125,87 & 535,831 &, 035 &, 678 \\
3 & 126,81 & 460,828 &, 384 &, 628 \\
4 & 135,78 & 486,306 &, 283 &, 645 \\
5 & 121,36 & 527,067 &, 066 &, 677 \\
6 & 136,53 & 493,011 &, 330 &, 640 \\
7 & 135,98 & 497,006 &, 311 &, 642 \\
8 & 128,10 & 467,665 &, 254 &, 654 \\
9 & 124,43 & 467,428 &, 355 &, 633 \\
10 & 133,24 & 463,507 &, 354 &, 633 \\
11 & 127,77 & 478,745 &, 369 &, 632 \\
12 & 128,60 & 457,003 &, 349 &, 633 \\
\hline
\end{tabular}

Alpha $=, 662 \quad$ * ritc $\geq .20 \mathrm{~N}=324$

\section{Validez de constructo}

Las correlaciones obtenidas entre las escalas mediante el coeficiente de correlación producto-momento de Pearson muestran hasta qué punto las variaciones o cambios en un ítem corresponden a las variaciones o cambios de la escala a la que pertenecen. Dichas correlaciones se presentan en la Tabla N. ${ }^{\circ}$. De las 71 correlaciones posibles entre los ítemes y escalas, tenemos 68 correlaciones estadísticamente significativas $(\mathrm{p}<.05)$, siendo todas ellas positivas. Las correlaciones estadísticamente significativas halladas alcanzan coeficientes que oscilan entre 0,42 y 0,70 . Existen 3 correlaciones menores a 0,4 (ítems 23, 69 y 71), las cuales no serán incluidas en el test final.

\section{Elaboración de baremos}

En el Anexo 1 se presentan las tablas con los baremos elaborados mediante el método de percentiles. Estos corresponden a los baremos normativos en percentiles (Pc) para la conversión de puntajes directos (PD) por grado de estudio y sexo, en la puntuación total obtenida en el Cuestionario de Estilos de Atributivos. 
Tabla $\mathbf{N}^{\circ}$ 4. Índice de correlación escala Ítem-Escala del Cuestionario de Estilos Atributivos.

\begin{tabular}{lcccccccc}
\hline \multirow{2}{*}{ Escala 1 } & item20 & item33 & item40 & item51 & item52 & item56 & item62 & item64 \\
&, 465 &, 615 &, 576 &, 422 &, 548 &, 495 &, 457 &, 554 \\
\hline \multirow{2}{*}{ Escala 2 } & item7 & item17 & item26 & item34 & item 47 & & & \\
&, 609 &, 633 &, 634 &, 538 &, 619 & & & \\
\hline \multirow{2}{*}{ Escala 3 } & item2 & item14 & item23 & item44 & item59 & item60 & & \\
&, 623 &, 632 &, 380 &, 605 &, 627 &, 656 & & \\
\hline \multirow{2}{*}{ Escala 4 } & item6 & item9 & item28 & item36 & item53 & item69 & \\
&, 513 &, 610 &, 673 &, 672 &, 637 &, 374 & \\
\hline \multirow{2}{*}{ Escala 5 } & item3 & item13 & item21 & item42 & item57 & item63 & \\
&, 466 &, 650 &, 578 &, 703 &, 667 &, 666 & \\
\hline \multirow{2}{*}{ Escala 6 } & item5 & item11 & item30 & item37 & item65 & & \\
&, 524 &, 568 &, 524 &, 672 &, 593 & & \\
\hline \multirow{2}{*}{ Escala 7 } & item8 & item19 & item49 & item68 & & & \\
&, 631 &, 609 &, 587 &, 591 & & & \\
\hline \multirow{2}{*}{ Escala 8 } & item10 & item16 & item27 & item29 & item54 & item70 & item71 \\
&, 636 &, 620 &, 471 &, 525 &, 582 &, 565 &, 381 & \\
\hline \multirow{2}{*}{ Escala 9 } & item4 & item12 & item31 & item38 & item55 & item66 & \\
&, 473 &, 554 &, 583 &, 568 &, 653 &, 632 & \\
\hline \multirow{2}{*}{ Escala 10 } & item1 & item15 & item24 & item41 & item45 & item61 & \\
&, 611 &, 508 &, 635 &, 561 &, 601 &, 547 & \\
\hline \multirow{2}{*}{ Escala 11 } & item18 & item25 & item39 & item48 & item67 & & \\
&, 549 &, 567 &, 700 &, 682 &, 514 & & \\
\hline \multirow{2}{*}{ Escala 12 } & item22 & item32 & item35 & item43 & item46 & item50 & item58 \\
&, 457 &, 446 &, 494 &, 578 &, 542 &, 522 &, 527 \\
\hline
\end{tabular}

\section{DISCUSIÓN}

Este resultado valida la importancia de la atribución causal en la motivación y, al mismo tiempo, constituye una justificación para la teoría atribucional de Weiner, que fundamenta la construcción del cuestionario inmerso en la teoría de la motivación.

De acuerdo con los objetivos de investigación, se analizó la validez y confiabilidad de la adaptación del Cuestionario de Estilos Atributivos, para determinar si funcionaba de forma apropiada en un grupo de estudiantes limeños de 4to y 5to año de secundaria. Los resultados encontrados en el análisis psicométrico aportan evidencia a favor de la adaptación del Cuestionario de Estilos Atributivos, obteniendo niveles de validez y confiabilidad aceptables, a partir de los cuales se puede concluir que la prueba puede funcionar en nuestro medio y es consistente.

Para calcular la validez del instrumento, se observó que mediante el criterio de jueces se obtuvo una $\mathrm{V}$ de Aiken, que solo rechazó el Item $72(\mathrm{~V}=0,50)$, estableciéndose la validez del instrumento. En cuanto a la confiabilidad del cuestionario, las correlaciones ítem-test 
oscilan entre 0,25 y 0,51 observándose que todos los bloques obtienen correlaciones significativas con el puntaje total de módulo, siendo las escalas más discriminativas la escala 1, Externalizacion e incontrolabilidad de los resultados académicos $(0,51)$, y la escala escala 3, Atribución del éxito académico a la habilidad $(0,84)$. El alpha de Cronbach calculado para el Cuestionario alcanza un valor de 0,662 de manera que se puede decir que el Cuestionario de Estilos Atributivos nos permite obtener puntajes confiables.

\section{CONCLUSIONES}

1. El Cuestionario de Estilos Atributivos resulta válido por los métodos de contenido y constructo.

2. El Cuestionario de Estilos Atributivos ha demostrado confiabilidad en la medición por los métodos de consistencia interna por la fórmula de Cronbach y por el método de correlación ítem test.

\section{REFERENCIAS BIBLIOGRÁFICAS}

1. Alonso, J. (1983a). Atribución de la causalidad y motivación de logro: Estudio evolutivo de la formación de juicios de atribución y de la influencia de las atribuciones en la motivación de logro. Tesis doctoral no publicada. Madrid: Universidad Autónoma de Madrid.

2. Alonso, J. (1983b). Atribución de la causalidad y motivación de logro. I: Estudio evolutivo de la utilización de información en la formación de juicios de atribución. Estudios de Psicología, 16, 13-27.

3. Alonso, J. (1984a). Atribución de la causalidad y motivación de logro. II: Estudio de la influencia de las atribuciones en el nivel manifiesto de motivación de logro. Estudios de Psicología, 17, 27-37.

4. Alonso, J. (1984b). Atribución de la causalidad y motivación de logro desde una perspectiva evolutiva. Evidencia empírica. Infancia y Aprendizaje, 26, 31-46.

5. Alonso, J., y Sánchez, J. (1986). Evaluación de los estilos atributivos en sujetos del Ciclo Superior de EGB. El cuestionario EAT. En J. Alonso Tapia (Dir.): Entrenamiento cognitivo y enriquecimiento motivacional. Vol.3. Informe final, CIDE. Madrid.

6. lonso, J. (1987). Análisis de las relaciones entre motivación de logro, estilos atributivos y expectativas de control a partir del estudio de la validez de constructo de los cuestionarios MAPE, EAT y ECO. Estudios de Psicología, 30, 45-69.

7. Alonso, J. (1991). Motivación y aprendizaje en el aula: Cómo enseñar a pensar. Madrid: Santillana.

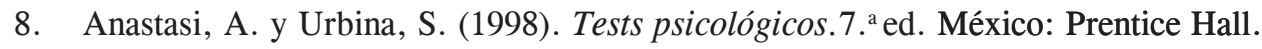


9. Ezcurra, L. (1988). Cuantificación de la Validez de Contenido por Criterio de Jueces. Revista de Psicología. Vol. VI. N. ${ }^{\circ}$ 1-2: 103-111.

10. Hernández, R y otros (2003). Metodología de la investigación. México: Mc Graw Hill Interamericana S.A.

11. Weiner, B. (1974). "An attributional interpretation of expectancy-value theory". B. Weiner (Ed.), Cognitive views of human motivation. New York, USA: Academic Press.

12. Weiner, B. (1985). "Spontaneous' causal thinking”. Psychological Bulletin, 97, 74-84.

13. Weiner, B. (1986). An attributional theory of motivation and emotion. New York: Springer-Verlag.

14. Weiner, B. (1992). Human motivation: Metaphors, Theories, and Research. Sage Publications.

15. Weiner, B. (2006). Social motivation, justice and the moral emotions. Mahwah, N.J: Erlbaum. 
Anexo 1. Rangos percentiles del CEA, para alumnos de educación secundaria de colegios estatales, según el grado de estudio y el sexo.

\begin{tabular}{|c|c|c|c|c|c|c|c|c|c|}
\hline \multirow{2}{*}{\multicolumn{2}{|c|}{ Total }} & \multicolumn{4}{|c|}{ Grado de estudio } & \multicolumn{4}{|c|}{ Sexo } \\
\hline & & \multicolumn{2}{|c|}{ 4to } & \multicolumn{2}{|c|}{ 5to } & \multicolumn{2}{|c|}{ Masculino } & \multicolumn{2}{|c|}{ Femenino } \\
\hline $\mathrm{PD}$ & $\mathrm{pc}$ & PD & $\mathrm{Pc}$ & $\mathrm{PD}$ & $\mathrm{Pc}$ & PD & $\mathrm{Pc}$ & PD & $\mathrm{Pc}$ \\
\hline 81 & 1 & 67 & 1 & 89 & 1 & 89 & 1 & 67 & 1 \\
\hline 84 & 1 & 81 & 1 & 94 & 1 & 94 & 1 & 81 & 1 \\
\hline 85 & 1 & 84 & 2 & 95 & 2 & 95 & 2 & 84 & 2 \\
\hline 89 & 2 & 85 & 3 & 96 & 3 & 96 & 4 & 85 & 3 \\
\hline 90 & 2 & 90 & 3 & 97 & 4 & 99 & 5 & 90 & 3 \\
\hline 94 & 2 & 94 & 4 & 99 & 7 & 102 & 6 & 94 & 4 \\
\hline 95 & 3 & 95 & 4 & 102 & 7 & 104 & 7 & 96 & 6 \\
\hline 96 & 5 & 96 & 6 & 105 & 8 & 106 & 7 & 97 & 7 \\
\hline 97 & 5 & 98 & 7 & 106 & 9 & 108 & 9 & 98 & 8 \\
\hline 98 & 6 & 102 & 8 & 107 & 9 & 109 & 10 & 99 & 8 \\
\hline 99 & 7 & 104 & 8 & 108 & 10 & 113 & 12 & 102 & 9 \\
\hline 102 & 7 & 106 & 9 & 109 & 12 & 114 & 12 & 105 & 10 \\
\hline 104 & 8 & 108 & 11 & 113 & 13 & 115 & 13 & 106 & 10 \\
\hline 105 & 8 & 109 & 12 & 115 & 13 & 116 & 15 & 107 & 11 \\
\hline 106 & 9 & 110 & 13 & 116 & 15 & 117 & 16 & 108 & 13 \\
\hline 107 & 9 & 111 & 14 & 117 & 16 & 118 & 18 & 109 & 14 \\
\hline 108 & 11 & 112 & 15 & 118 & 16 & 119 & 20 & 110 & 15 \\
\hline 109 & 12 & 113 & 16 & 119 & 20 & 120 & 21 & 111 & 16 \\
\hline 110 & 13 & 114 & 18 & 120 & 21 & 121 & 23 & 112 & 17 \\
\hline 111 & 13 & 116 & 19 & 121 & 24 & 122 & 24 & 114 & 19 \\
\hline 112 & 14 & 117 & 20 & 122 & 25 & 123 & 24 & 116 & 19 \\
\hline 113 & 15 & 118 & 22 & 123 & 27 & 124 & 26 & 117 & 20 \\
\hline 114 & 15 & 119 & 26 & 124 & 30 & 125 & 27 & 119 & 25 \\
\hline 115 & 16 & 120 & 27 & 125 & 32 & 126 & 28 & 120 & 27 \\
\hline 116 & 17 & 121 & 29 & 126 & 34 & 127 & 29 & 121 & 30 \\
\hline 117 & 18 & 122 & 29 & 127 & 34 & 128 & 31 & 122 & 31 \\
\hline 118 & 19 & 123 & 30 & 128 & 38 & 129 & 32 & 123 & 34 \\
\hline 119 & 23 & 124 & 33 & 129 & 39 & 130 & 36 & 124 & 38 \\
\hline 120 & 24 & 125 & 34 & 130 & 42 & 131 & 40 & 125 & 39 \\
\hline 121 & 27 & 127 & 34 & 131 & 44 & 132 & 41 & 126 & 40 \\
\hline 122 & 27 & 128 & 36 & 132 & 45 & 133 & 45 & 128 & 43 \\
\hline 123 & 29 & 129 & 38 & 133 & 47 & 134 & 47 & 129 & 45 \\
\hline 124 & 32 & 130 & 43 & 134 & 49 & 135 & 50 & 130 & 49 \\
\hline 125 & 33 & 131 & 45 & 135 & 51 & 136 & 51 & 131 & 50 \\
\hline 126 & 34 & 132 & 47 & 136 & 54 & 137 & 52 & 132 & 51 \\
\hline 127 & 34 & 133 & 49 & 137 & 54 & 138 & 54 & 133 & 52 \\
\hline 128 & 37 & 134 & 50 & 138 & 56 & 139 & 57 & 134 & 52 \\
\hline 129 & 38 & 135 & 53 & 139 & 60 & 140 & 59 & 135 & 55 \\
\hline
\end{tabular}


María Matalinares C., Rosa Tueros C., Juan Yaringaño L..

\begin{tabular}{|c|c|c|c|c|c|c|c|c|c|}
\hline \multirow{2}{*}{\multicolumn{2}{|c|}{ Total }} & \multicolumn{4}{|c|}{ Grado de estudio } & \multicolumn{4}{|c|}{ Sexo } \\
\hline & & \multicolumn{2}{|c|}{ 4to } & \multicolumn{2}{|c|}{ 5to } & \multicolumn{2}{|c|}{ Masculino } & \multicolumn{2}{|c|}{ Femenino } \\
\hline PD & $\mathrm{pc}$ & PD & $\mathrm{Pc}$ & PD & $\mathrm{Pc}$ & PD & Pc & PD & $\mathrm{Pc}$ \\
\hline 130 & 42 & 136 & 57 & 140 & 61 & 141 & 60 & 136 & 59 \\
\hline 131 & 44 & 137 & 58 & 142 & 62 & 142 & 62 & 137 & 60 \\
\hline 132 & 46 & 138 & 59 & 144 & 64 & 143 & 62 & 138 & 61 \\
\hline 133 & 48 & 139 & 61 & 145 & 66 & 144 & 64 & 139 & 65 \\
\hline 134 & 49 & 140 & 63 & 146 & 68 & 145 & 66 & 140 & 66 \\
\hline 135 & 52 & 141 & 66 & 147 & 70 & 146 & 67 & 141 & 66 \\
\hline 136 & 55 & 142 & 68 & 148 & 72 & 147 & 69 & 142 & 68 \\
\hline 137 & 56 & 143 & 69 & 149 & 74 & 148 & 70 & 143 & 70 \\
\hline 138 & 58 & 144 & 71 & 150 & 76 & 149 & 72 & 144 & 71 \\
\hline 139 & 60 & 145 & 72 & 151 & 79 & 150 & 75 & 145 & 73 \\
\hline 140 & 62 & 146 & 73 & 152 & 80 & 151 & 77 & 146 & 74 \\
\hline 141 & 63 & 147 & 74 & 153 & 80 & 152 & 78 & 147 & 75 \\
\hline PD & $\mathrm{pc}$ & PD & $\mathrm{Pc}$ & PD & $\mathrm{Pc}$ & PD & $\mathrm{Pc}$ & $\mathrm{PD}$ & $\mathrm{Pc}$ \\
\hline 142 & 65 & 148 & 74 & 154 & 81 & 154 & 78 & 148 & 76 \\
\hline 143 & 66 & 149 & 76 & 155 & 82 & 155 & 79 & 149 & 77 \\
\hline 144 & 68 & 150 & 77 & 156 & 84 & 156 & 81 & 150 & 78 \\
\hline 145 & 69 & 152 & 79 & 157 & 85 & 157 & 82 & 151 & 79 \\
\hline 146 & 70 & 153 & 79 & 158 & 87 & 158 & 84 & 152 & 81 \\
\hline 147 & 72 & 154 & 81 & 162 & 88 & 162 & 86 & 153 & 83 \\
\hline 148 & 73 & 155 & 81 & 164 & 88 & 164 & 87 & 154 & 84 \\
\hline 149 & 75 & 156 & 83 & 165 & 89 & 165 & 88 & 155 & 85 \\
\hline 150 & 76 & 157 & 85 & 168 & 90 & 166 & 89 & 156 & 86 \\
\hline 151 & 78 & 158 & 86 & 170 & 91 & 168 & 90 & 157 & 89 \\
\hline 152 & 79 & 160 & 88 & 171 & 92 & 170 & 91 & 158 & 90 \\
\hline 153 & 80 & 162 & 89 & 172 & 94 & 171 & 92 & 160 & 91 \\
\hline 154 & 81 & 164 & 90 & 173 & 95 & 172 & 93 & 164 & 92 \\
\hline 155 & 82 & 165 & 91 & 174 & 96 & 174 & 93 & 165 & 92 \\
\hline 156 & 84 & 166 & 93 & 177 & 96 & 177 & 95 & 166 & 93 \\
\hline 157 & 85 & 170 & 93 & 179 & 98 & 180 & 95 & 170 & 94 \\
\hline 158 & 87 & 172 & 94 & 180 & 98 & 181 & 96 & 172 & 95 \\
\hline 160 & 87 & 177 & 94 & 185 & 99 & 183 & 96 & 173 & 95 \\
\hline 162 & 88 & 178 & 95 & 188 & 100 & 185 & 97 & 174 & 96 \\
\hline 164 & 89 & 181 & 96 & & & 186 & 98 & 178 & 97 \\
\hline 165 & 90 & 183 & 96 & & & 188 & 98 & 179 & 98 \\
\hline 166 & 91 & 186 & 97 & & & 192 & 99 & 185 & 99 \\
\hline 168 & 91 & 188 & 98 & & & 202 & 99 & 188 & 99 \\
\hline 170 & 92 & 192 & 98 & & & & & & \\
\hline 171 & 93 & 202 & 99 & & & & & & \\
\hline 172 & 94 & 205 & 99 & & & & & & \\
\hline 173 & 94 & & & & & & & & \\
\hline
\end{tabular}




\begin{tabular}{|c|c|c|c|c|c|c|c|c|c|}
\hline \multirow{2}{*}{\multicolumn{2}{|c|}{ Total }} & \multicolumn{4}{|c|}{ Grado de estudio } & \multicolumn{4}{|c|}{ Sexo } \\
\hline & & \multicolumn{2}{|c|}{ 4to } & \multicolumn{2}{|c|}{ 5to } & \multicolumn{2}{|c|}{ Masculino } & \multicolumn{2}{|c|}{ Femenino } \\
\hline PD & $\mathrm{pc}$ & PD & Pc & PD & Pc & $\mathrm{PD}$ & $\mathrm{Pc}$ & PD & $\mathrm{Pc}$ \\
\hline 174 & 95 & & & & & & & & \\
\hline 177 & 95 & & & & & & & & \\
\hline 178 & 96 & & & & & & & & \\
\hline 179 & 96 & & & & & & & & \\
\hline 180 & 97 & & & & & & & & \\
\hline 181 & 97 & & & & & & & & \\
\hline 183 & 97 & & & & & & & & \\
\hline 185 & 98 & & & & & & & & \\
\hline 186 & 98 & & & & & & & & \\
\hline 188 & 99 & & & & & & & & \\
\hline 192 & 99 & & & & & & & & \\
\hline 202 & 99 & & & & & & & & \\
\hline
\end{tabular}

\section{CUESTIONARIO DE ESTILOS ATRIBUTIVOS}

\section{INSTRUCCIONES}

En este cuadernillo encontrarás una serie de afirmaciones sobre las causas del éxito y el fracaso en distintas situaciones, en las cuales debes señalar tu grado de ACUERDO o DESACUERDO. No hay contestaciones correctas o incorrectas, ya que las personas podemos considerar las cosas desde distintos puntos de vista.

Contesta con sinceridad de modo que se pueda conocer mejor tu forma de ser.

Anota tus respuestas en la Hoja de Respuestas que te han entregado. Señala tu grado de ACUERDO o DESACUERDO con el contenido de cada afirmación marcando con una X en la escala correspondiente. Por ejemplo en la afirmación numero 20:

20. Para mí aprobar un examen es como si me tocase la lotería.

Puedes elegir 5 posibilidades

\begin{tabular}{|l|l|l|l|l|l|}
\hline 20. & $\mathbf{0}$ & $\mathbf{1}$ & $\mathbf{2}$ & $\mathbf{3}$ & $\mathbf{4}$ \\
\hline
\end{tabular}

Ten presente que:

Una puntuación de 0 significa Máximo Desacuerdo o Totalmente en desacuerdo, y

Una puntuación de 4 significa Máximo Acuerdo o Totalmente de acuerdo 


\section{RECUERDA QUE DEBES CONTESTAR SINCERAMENTE YA QUE, EN CASO CONTRARIO, LA INFORMACIÓN OBTENIDA NO SERVIRÁ PARA TU ORIENTACIÓN PSICOLÓGICA}

Espera a que se dé la señal para empezar.

1. Frecuentemente mi buena suerte ha sido la causa de que me haya llevado bien con los profesores.

2. Pienso que mis buenas notas reflejan, sobre todo, lo inteligente que soy para los estudios.

3. En mi caso, sacar buenas notas se ha debido, a mi propio esfuerzo.

4. Si he conseguido llevarme bien con mis padres se ha debido al esfuerzo que he puesto en comprenderlos.

5. Por lo general, si he obtenido malos resultados en lenguaje creo que ha sido por mala suerte.

6. Mis malas notas en Lenguaje se han debido, con frecuencia, a que el profesor pone bajas notas

7. Si alguna vez he obtenido bajas calificaciones en Matemáticas se ha debido a falta de esfuerzo.

8. Si jalo un curso, probablemente es por no estar capacitado intelectualmente.

9. Con frecuencia mis malas notas se deben a que el profesor no está bien preparado para enseñarme.

10. Si tengo pocos amigos es por mi falta de habilidad para tratarles.

11. Mi mala suerte ha sido muchas veces lo que hizo que no tuviese mejores notas.

12. Si consigo hacer amistades duraderas es por mi esfuerzo en ser amable, paciente y tolerante.

13. Siempre que he obtenido buenas notas ha sido porque he estudiado mucho

14. Si saco buenas notas es por mi buena capacidad para los estudios.

15. La mayoría de las veces en que he conseguido entablar amistades ha sido por casualidad.

16. Si en algún momento de mi vida me he encontrado sin amigos ha sido porque no me he esforzado en buscarlos.

17. En general, las notas bajas en Lenguaje han reflejado mi poco trabajo y esfuerzo.

18. Si tengo amigos puedo decir que es gracias a mi diplomacia, a que se como llevarme bien

19. Si sacase malas notas pensaría que no tengo el talento necesario para comprender esos cursos.

20. La suerte ha sido, por lo general, la causa de mis buenas notas en Lenguaje.

21. Cuando he trabajado con empeño, generalmente he podido superar los obstáculos que me impedían tener éxito en los estudios.

22. La mayoría de las veces, si mis relaciones con los demás no han ido bien, ha sido porque ellos no se han esforzado en conocerme realmente.

23. Cosas que han ocurrido por casualidad han sido con frecuencia las que me han ayudado a llevarme bien con mis padres. 
24. Cuando he podido entenderme con algún profesor se ha debido, sobre todo, a mi capacidad para el trato con los demás.

25. Las bajas notas que he obtenido han sido, sobre todo, porque no me he esforzado lo suficiente.

26. Por mi propia experiencia sé que si no me he llevado bien con algún profesor ha sido por mi poco esfuerzo en entablar amistad.

27. Si he tenido malas notas en Matemáticas a menudo ha sido porque el profesor explicaba mal.

28. Casi siempre la causa de que no haya caído bien a algún profesor ha sido mi torpeza en el trato.

29. Frecuentemente mis bajas notas en Matemáticas se han debido, sobre todo, a equivocaciones casuales.

30. En mi caso, si me he llevado bien con gente de mi edad pero del otro sexo, ha sido sobre todo por mi esfuerzo.

31. Malentendidos han sido, con frecuencia, los responsables de que mis relaciones con los demás hayan fracasado.

32. La suerte es, con frecuencia, el principal factor responsable de mi éxito en los estudios.

33. Normalmente, si recibo una mala nota en una asignatura es porque no he estudiado lo suficiente.

34. Mis dificultades con los amigos han comenzado, a menudo, con comentarios que, por casualidad, han sido mal interpretados.

35. A menudo, si he tenido malas notas ha sido porque los profesores no me han explicado bien las lecciones.

36. La casualidad ha sido la causa de que a veces haya tenido puntuaciones bajas.

37. En mi caso, el haber conseguido hacer una amistad ha dependido del empeño que haya puesto en ello.

38. Frecuentemente, gracias a mi habilidad para las relaciones he podido conseguir buenos amigos.

39. Con frecuencia, si he sacado buenas notas en una asignatura, ha sido porque el profesor daba puntuaciones altas con mucha facilidad.

40. Si tengo amigos es gracias a su iniciativa más que a la mía.

41. Si tengo buenas notas en Matemáticas es por el esfuerzo y empeño que pongo.

42. Me es casi imposible comprender por qué desagrado a algunas personas.

43. Mi inteligencia constituye el factor más importante a la hora de conseguir buenas notas.

44. En mi caso, si me he llevado bien con personas de mi edad pero del otro sexo, ha sido principalmente por suerte.

45. Haga lo que haga, hay gente a la que no le caigo bien.

46. Las malas notas significan para mí que no he trabajado con suficiente empeño.

47. Cuando he podido entenderme con alguien se ha debido a mi facilidad para el trato con las personas.

48. Si sacase malas notas dudaría de mi inteligencia.

49. Si no he tenido amigos ha sido porque no me he encontrado con las personas adecuadas. 
50. Normalmente he sacado buenas notas, sólo porque lo que tenía que aprender, era fácil.

51. Por lo general, cuando he sacado buenas notas ha sido porque el examen tenía preguntas que había estudiado.

52. Normalmente, si he sacado malas notas ha sido porque el profesor era un tacaño al puntuar.

53. Con frecuencia, cuando me he encontrado sólo ha sido por no saber tratar con los amigos.

54. Si he conseguido que mis amigos me duren ha sido porque me he esforzado en conseguirlo.

55. Con frecuencia mis malas notas se deben a la mala suerte de tocarme justamente las preguntas que no he preparado.

56. Cuando me he esforzado y he trabajado de firme, he conseguido buenas notas en Lenguaje.

57. La antipatía que algunos chicos/chicas parecen sentir por mí es la principal causa de que mis relaciones con ellos no marchen bien.

58. Si alguna vez he obtenido buenos resultados en Lenguaje, se ha debido a mi capacidad para ese curso

59. En general, cuando alguna vez he sacado buenas notas, ha sido principalmente por lo listo que soy.

60. Conseguir amigos no es cuestión de que yo quiera tenerlos: normalmente creo que mi éxito se debe a la suerte.

61. Por lo general, apruebo simplemente por suerte.

62. Normalmente, cuando he trabajado de firme ha conseguido tener éxito en los estudios.

63. Creo que, con frecuencia, cuando he obtenido buenas notas ha sido por la facilidad de los cursos.

64. Me parece que, normalmente, mis notas bajas se han debido a la casualidad.

65. Si alguna vez me he llevado bien con algún profesor, ha sido porque he puesto mucho interés en ello.

66. Haberme llevado bien con alguien ha sido cuestión de habilidad.

67. Cuando saco malas notas pienso que no soy inteligente

68. Es frecuente, si saco malas notas, que sea porque el profesor no ha hecho interesante la asignatura. 